\title{
Characteristics of Ischemic Versus Hemorrhagic Stroke in Patients Receiving Oral Anticoagulants: Results of the PASTA Study
}

\author{
Satoshi Suda ${ }^{1}$, Arata $\mathrm{Abe}^{2}$, Yasuyuki Iguchi ${ }^{3}$, Yoshiki Yagita ${ }^{4}$, Takao Kanzawa ${ }^{5}$, \\ Seiji Okubo ${ }^{6}$, Nobuyuki Ohara ${ }^{7}$, Takayuki Mizunari ${ }^{8}$, Mineo Yamazaki ${ }^{9}$, \\ Nobuhito Nakajima $^{10}$, Kimito Kondo ${ }^{11}$, Shigeru Fujimoto ${ }^{12}$, Takeshi Inoue ${ }^{13}$, \\ Takeshi Iwanaga $^{14}$, Yuka Terasawa ${ }^{15}$, Kensaku Shibazaki ${ }^{16}$, Yu Kono ${ }^{17}$, Makoto Nakajima ${ }^{18}$, \\ Masataka Nakajima $^{19}$, Masahiro Mishina ${ }^{20}$, Koji Adachi ${ }^{21}$, Ichiro Imafuku ${ }^{22}$, Koichi Nomura ${ }^{23}$, \\ Takehiko Nagao ${ }^{24}$, Hiroshi Yaguchi ${ }^{25}$, Sadahisa Okamoto ${ }^{26}$, \\ Masato Osaki ${ }^{27}$ and Kazumi Kimura ${ }^{1}$
}

\begin{abstract}
:
Objective Limited data exist regarding the comparative detailed clinical characteristics of patients with ischemic stroke (IS)/transient ischemic attack (TIA) and intracerebral hemorrhage (ICH) receiving oral anticoagulants (OACs).

Methods The prospective analysis of stroke patients taking oral anticoagulants (PASTA) registry, a multicenter registry of 1,043 stroke patients receiving OACs (vitamin K antagonists [VKAs] or non-vitamin K oral antagonists [NOACs]) across 25 medical institutions throughout Japan, was used. Univariate and multivariable analyses were used to analyze differences in clinical characteristics between IS/TIA and ICH patients with atrial fibrillation (AF) who were registered in the PASTA registry.

Results There was no significant differences in cardiovascular risk factors, such as hypertension, diabetes mellitus, dyslipidemia, smoking, or alcohol consumption (all $\mathrm{p}>0.05$ ), between IS/TIA and ICH among both NOAC and VKA users. Cerebral microbleeds (CMBs) (odds ratio [OR], 4.77; $\mathrm{p}<0.0001$ ) were independently associated with $\mathrm{ICH}$, and high brain natriuretic peptide/ $\mathrm{N}$-terminal pro B-type natriuretic peptide levels $(\mathrm{OR}$, 1.89; $\mathrm{p}=0.0390)$ were independently associated with IS/TIA among NOAC users. A history of ICH (OR, 13.59; $\mathrm{p}=0.0279)$ and the high prothrombin time-international normalized ratio (PT-INR) (OR, 1.17; $\mathrm{p}<$ $0.0001)$ were independently associated with ICH, and a history of IS/TIA (OR, 3.37; 95\% CI, 1.34-8.49; $\mathrm{p}=$ $0.0101)$ and high D-dimer levels (OR, 2.47; 95\% CI, 1.05-5.82; $\mathrm{p}=0.0377)$ were independently associated with IS/TIA among VKA users.
\end{abstract}

${ }^{1}$ Department of Neurology, Nippon Medical School, Japan, ${ }^{2}$ Department of Neurology and Stroke Medicine, Tokyo Metropolitan Tama Medical Center, Japan, ${ }^{3}$ Department of Neurology, The Jikei University School of Medicine, Japan, ${ }^{4}$ Department of Stroke Medicine, Kawasaki Medical School, Japan, ${ }^{5}$ Department of Stroke Medicine, Institute of Brain and Blood Vessels, Mihara Memorial Hospital, Japan, ${ }^{6}$ Department of Cerebrovascular Medicine, NTT Medical Center Tokyo, Japan, ${ }^{7}$ Department of Neurology, Kobe City Medical Center General Hospital, Japan, ${ }^{8}$ Department of Neurosurgery, Nippon Medical School Chiba Hokusoh Hospital, Japan, ${ }^{9}$ Department of Neurology, Nippon Medical School Chiba Hokusoh Hospital, Japan, ${ }^{10}$ Department of Neurology, Kitamurayama Hospital, Japan, ${ }^{11}$ Department of Neurology, Hokuto Hospital, Japan, ${ }^{12}$ Division of Neurology, Department of Medicine, Jichi Medical University Hospital, Japan, ${ }^{13}$ Department of Stroke Medicine, Kawasaki Medical School General Medical Center, Japan, ${ }^{14}$ Department of Stroke Medicine, Japanese Red Cross Okayama Hospital, Japan, ${ }^{15}$ Department of Neurology, Brain Attack Center Ota Memorial Hospital, Japan, ${ }^{16}$ Department of Stroke Medicine, Kurashiki Heisei Hospital, Japan, ${ }^{17}$ Department of Neurology, Fuji City General Hospital, Japan, ${ }^{18}$ Department of Neurology, Graduate School of Medical Sciences, Kumamoto University, Japan, ${ }^{19}$ Department of Neurology, Heisei-Tateishi Hospital, Japan, ${ }^{20}$ Department of Neuro-pathophysiological Imaging, Graduate School of Medicine, Nippon Medical School, Japan, ${ }^{21}$ Department of Neurological Surgery, Nippon Medical School Musashi-Kosugi Hospital, Japan, ${ }^{22}$ Department of Neurology, Yokohama Rosai Hospital, Japan, ${ }^{23}$ Department of Neurology, Shioda Hospital, Japan, ${ }^{24}$ Department of Neurology, Nippon Medical School Tama Nagayama Hospital, Japan, ${ }^{25}$ Department of Neurology, The Jikei University Kashiwa Hospital, Japan, ${ }^{26}$ Department of Neurology, Omuta Tenryo Hospital, Japan and ${ }^{27}$ Department of Cerebrovascular Medicine, Steel Memorial Yawata Hospital, Japan

Received: June 8, 2021; Accepted: July 20, 2021; Advance Publication by J-STAGE: September 4, 2021

Correspondence to Dr. Satoshi Suda, suda-sa@nms.ac.jp 
Conclusion The presence of CMBs, a history of stroke, natriuretic peptide and D-dimer levels, and PT-INR may be useful for risk stratification of either IS/TIA or ICH development in patients with AF receiving OACs.

Key words: atrial fibrillation, intracerebral hemorrhage, ischemic stroke, non-vitamin $\mathrm{K}$ antagonist, vitamin $\mathrm{K}$ antagonist

(Intern Med Advance Publication)

(DOI: 10.2169/internalmedicine.8113-21)

\section{Introduction}

Atrial fibrillation (AF)-related stroke and acute venous thromboembolism are associated with substantial morbidity and mortality and are increasing in prevalence in Japan (1-3). Vitamin K antagonists (VKAs) have been the cornerstone of therapy for the prevention of thromboembolism in patients with $\mathrm{AF}$ and deep vein thrombosis. However, while inexpensive, VKAs have a narrow therapeutic window, require frequent monitoring, and have many interactions with food and drugs, resulting in poor adherence (4). Nonvitamin $\mathrm{K}$ oral antagonists (NOACs) are confirmed to be as effective as VKAs and are associated with a lower risk of intracranial hemorrhage (5-7). Recent guidelines specify NOACs as first-line drugs for the prevention of embolism in patients with $\mathrm{AF}(8,9)$.

NOACs are prescribed liberally in clinical practice (10), and the incidences of ischemic stroke (IS)/transient ischemic attack (TIA) and intracerebral hemorrhage (ICH) related to NOACs are expected to increase. Stroke prevention using OACs must balance the benefit of reducing the risk of IS against the increased risk of major bleeding, including ICH. A better distinction between patients who are primarily at risk of experiencing either IS/TIA or ICH is desirable, but the criteria of the most widely used clinical risk scores for thromboembolism and bleeding overlap considerably $(11,12)$. Furthermore, the recommended INR values for VKA and the criteria for NOAC dosing differ between Japan and Western countries (13). Previous studies showing the characteristics or outcomes of patients with stroke who received OACs were predominantly retrospective, singlecenter, relatively small-sample studies that enrolled patients with IS and ICH separately (14-18). Therefore, we established the multicenter PASTA registry to support current research on the status of stroke in patients receiving OACs in Japan (19).

The present study is the first to analyze the PASTA registry data, aiming to clarify the differences in clinical characteristics between IS/TIA and ICH patients with AF who are receiving NOACs and VKAs.

\section{Materials and Methods}

\section{Standard protocol approvals, registrations, and pa- tient consent}

This investigator-initiated, multicenter, prospective, cohort study utilized the PASTA registry as previously reported (19). IS, TIA, and ICH patients receiving OACs were prospectively enrolled across 25 medical institutions throughout Japan between April 2016 and September 2019. Patients were divided into the IS/TIA and ICH groups.

This study was approved by the ethics committee of Nippon Medical School and conformed to the tenets of the Declaration of Helsinki. All participants or their family members provided their written informed consent prior to study participation.

\section{Clinical characteristics}

We collected data on clinical characteristics, including the sex, age, cardiovascular risk factors, pre-morbid modified Rankin scale (mRS) score, and pre-stroke $\mathrm{CHADS}_{2}, \mathrm{CHA}_{2}$ $\mathrm{DS}_{2}$-VASc, or HAS-BLED score. Cardiovascular risk factors were defined as (1) hypertension: history of using antihypertensive agents, systolic blood pressure $\geq 140 \mathrm{~mm} \mathrm{Hg}$, or diastolic blood pressure $\geq 90 \mathrm{~mm} \mathrm{Hg}$ before or $\geq 2$ weeks after stroke onset; (2) diabetes mellitus: use of hypoglycemic agents, random glucose level $\geq 200 \mathrm{mg} / \mathrm{dL}$, or glycosylated hemoglobin $\geq 6.0 \%$ on admission; (3) hyperlipidemia: use of antihyperlipidemic agents, or a serum total cholesterol level $\geq 220 \mathrm{mg} / \mathrm{dL}$; and (4) current smoker. The prestroke $\mathrm{CHADS}_{2}$ or $\mathrm{CHA}_{2} \mathrm{DS}_{2}$ VASc score was calculated for each patient based on the published guideline (20). The blood pressure (BP) status one month before the onset of stroke was also recorded. A poor BP control was defined as a systolic BP $\geq 140 \mathrm{mmHg}$ and/or diastolic BP $\geq 90 \mathrm{mmHg}$ with or without pre-stroke antihypertensive medication. The presence of an abnormal renal function, abnormal liver function, and alcohol intake defined by the HAS-BLED score was also evaluated $(20,21)$.

Routine blood biochemistry examinations were performed on admission. A high D-dimer level was defined as $>1.0 \mu \mathrm{g} /$ $\mathrm{mL}$ (22-24). High brain natriuretic peptide (BNP) or $\mathrm{N}$ terminal pro B-type natriuretic peptide (NT-proBNP) levels were defined as $>100$ or $>300 \mathrm{pg} / \mathrm{mL}$, respectively $(25,26)$. 


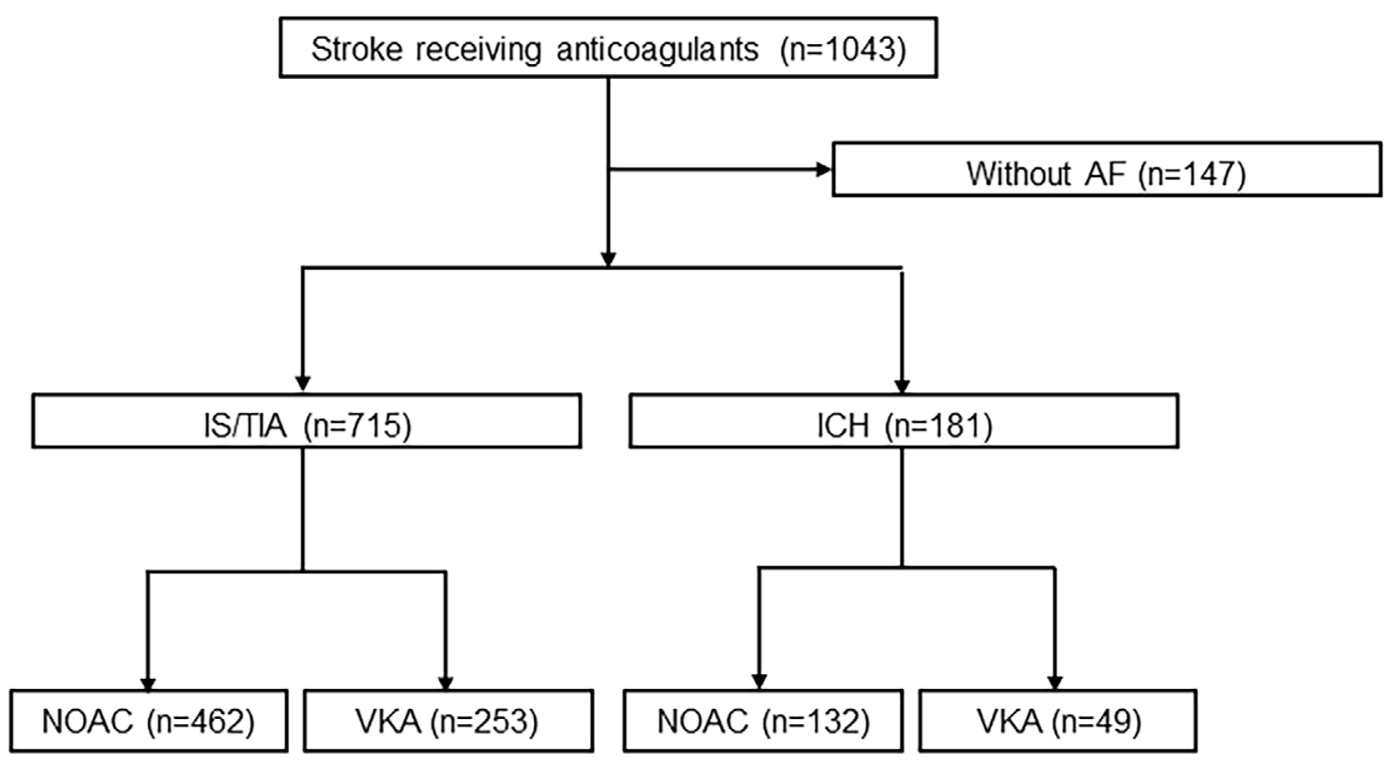

Figure 1. Study flowchart. ICH: intracerebral hemorrhage, IS: ischemic stroke, NOAC: non-vitamin $K$ oral antagonist, TIA: transient ischemic attack, VKA: vitamin K antagonist

Gradient-recalled echo T2*-weighted images were assessed for evidence of cerebral microbleeds (CMBs), defined as parenchymal hemorrhage $\leq 10 \mathrm{~mm}$ in diameter (27). Stroke severity on admission and on discharge was assessed using the National Institutes of Health Stroke Scale (NIHSS) score and the mRS score, respectively.

\section{Statistical analyses}

We roughly compared the clinical characteristics between the IS/TIA and ICH groups, and then according to prior NOAC or VKA use. Univariate analyses were performed using the chi-squared test or Wilcoxon's rank-sum test. Data are presented as medians (interquartile range) or numbers (\%). A multivariable logistic regression analysis was performed to identify independent factors associated with an increased incidence of ICH or IS/TIA. Sex, age, and all clinical characteristics with $\mathrm{p}<0.05$ in the univariate analyses were entered into the model. The $\mathrm{CHADS}_{2}, \mathrm{CHA}_{2} \mathrm{DS}_{2}-\mathrm{VASc}$, and HAS-BLED scores were excluded due to variable duplication, and the initial NIHSS score was excluded because these parameters were consequences of stroke. A two-tailed $P$-value of $<0.05$ was considered significant. Analyses were performed using the JMP version 13 statistical software program (SAS Institute Inc., Cary, NC, USA).

\section{Results}

\section{Differences in clinical characteristics between ISITIA and $\mathrm{ICH}$}

A total of 1,043 patients with IS/TIA or ICH (women, 415 patients; median age, 79 [interquartile range, 72-84] years old; and NIHSS score, 6 [interquartile range, 2-18]) were enrolled in the PASTA study. The final cohort for the present analysis comprised 896 patients (Fig. 1). There were
$715(79.8 \%)$ and $181(20.2 \%)$ patients in the IS/TIA and ICH groups, respectively. Among IS/TIA patients, NOACs and VKAs were prescribed in 462 (64.6\% [dabigatran, $n=$ 67; rivaroxaban, $n=139$; apixaban, $n=143$; or edoxaban, $n=$ 113]) and $253(35.4 \%)$, respectively. Among ICH patients, NOACs and VKAs were prescribed in 132 (62.9\% [dabigatran, $n=4$; rivaroxaban, $n=47$; apixaban, $n=46$; or edoxaban, $\mathrm{n}=35])$ and $49(37.1 \%)$, respectively.

Table 1 presents the clinical characteristics of both groups. IS/TIA patients were older than ICH patients $(\mathrm{p}<$ $0.0001)$. Male sex $(p=0.0456)$, prior NOAC plus antiplatelet therapy $(\mathrm{p}=0.0447)$, a history of ICH $(\mathrm{p}<0.0001)$, poor prestroke BP control $(p<0.0001)$, alcohol use $(p=0.0141)$, and CMBs $(\mathrm{p}<0.0001)$ were more prevalent among ICH patients than among IS/TIA patients. The Ccr $(\mathrm{p}<0.0001)$, glycated hemoglobin A1c (HbA1c) level $(p=0.0268)$, APTT $(p=$ $0.0254)$, NIHSS score on admission $(\mathrm{p}<0.0001), \mathrm{mRS}$ score on discharge $(\mathrm{p}<0.0001)$, and in-hospital mortality $(\mathrm{p}=$ 0.0012) were also significantly higher among ICH patients than among IS/TIA patients. The prevalence of comorbidities associated with stroke, such as hypertension, diabetes mellitus, dyslipidemia, and smoking, and HAS-BLED scores did not significantly differ between both groups (all $\mathrm{p}>0.05$ ). Furthermore, the $\mathrm{CHADS}_{2}(\mathrm{p}=0.0123)$ and $\mathrm{CHA}_{2} \mathrm{DS}_{2}-\mathrm{VASc}$ $(\mathrm{p}=0.0011)$ scores and the $\mathrm{D}$-dimer $(\mathrm{p}<0.0001)$ and $\mathrm{BNP} /$ NT-proBNP levels $(\mathrm{p}=0.0005)$ were higher among IS/TIA patients than $\mathrm{ICH}$ patients.

\section{Factors associated with ICH or IS/TIA}

Table 2 presents the findings of the multivariable logistic regression analysis of factors associated with $\mathrm{ICH}$. A history of ICH (odds ratio [OR], 4.03; 95\% confidence interval [CI], 1.64-9.92; p=0.0024), a high APTT (OR, 1.02; 95\% $\mathrm{CI}$, 1.00-1.04; $\mathrm{p}=0.0317$ ), and CMBs (OR, 3.64; 95\% CI, 2.25-5.87; $\mathrm{p}<0.0001)$ were independently associated with 
Table 1. Comparison of Clinical Characteristics between IS/TIA and ICH.

\begin{tabular}{|c|c|c|c|c|}
\hline Variable & $\begin{array}{c}\text { Total } \\
\mathrm{n}=896\end{array}$ & $\begin{array}{l}\text { IS/TIA } \\
\mathrm{n}=715\end{array}$ & $\begin{array}{c}\text { ICH } \\
n=181\end{array}$ & $\mathrm{p}$ value \\
\hline Age, years, median (IQR) & $79(73-84)$ & $80(74-85)$ & $77(70-82)$ & $<0.0001$ \\
\hline Female gender, $\mathrm{n}(\%)$ & $345(38.5)$ & $287(40.1)$ & $58(32.0)$ & 0.0456 \\
\hline \multicolumn{5}{|l|}{ Prior antithrombotic therapy, $\mathrm{n}(\%)$} \\
\hline NOAC & $510(56.9)$ & $402(56.2)$ & $108(59.7)$ & 0.4031 \\
\hline NOAC plus APT & $84(9.4)$ & $60(8.4)$ & $24(13.3)$ & 0.0447 \\
\hline VKA & $233(26.0)$ & $194(27.1)$ & $39(21.6)$ & 0.1259 \\
\hline VKA plus APT & $69(7.7)$ & $59(8.3)$ & $10(5.5)$ & 0.2190 \\
\hline \multicolumn{5}{|l|}{ Risk factors, n (\%) } \\
\hline Previous IS/TIA & $388(43.3)$ & $319(44.6)$ & $69(38.1)$ & 0.1152 \\
\hline Previous ICH & $33(3.7)$ & $14(1.96)$ & $19(10.5)$ & $<0.0001$ \\
\hline Hypertension & $721(80.5)$ & $569(79.6)$ & $152(84.0)$ & 0.1825 \\
\hline Diabetes mellitus & $243(27.1)$ & $201(28.1)$ & $42(23.2)$ & 0.1846 \\
\hline Congestive heart failure & $248(27.7)$ & $202(28.3)$ & $46(25.4)$ & 0.4460 \\
\hline Dyslipidemia & $323(36.1)$ & $260(36.4)$ & $63(34.8)$ & 0.6967 \\
\hline Smoking & $209(23.3)$ & $168(23.5)$ & $41(22.7)$ & 0.8103 \\
\hline Alcohol & $166(18.5)$ & $121(16.9)$ & $45(24.9)$ & 0.0141 \\
\hline History of vascular disease, $\mathrm{n}(\%)$ & $129(14.4)$ & $103(14.4)$ & $26(14.4)$ & 0.9888 \\
\hline Abnormal renal function, $\mathrm{n}(\%)$ & $24(2.7)$ & $18(2.5)$ & $6(3.3)$ & 0.5528 \\
\hline Abnormal liver function, $\mathrm{n}(\%)$ & $25(2.8)$ & $16(2.2)$ & $9(5.0)$ & 0.0460 \\
\hline Poor BP control prior to admission, $\mathrm{n}(\%)$ & $171(19.6)$ & $119(17.0)$ & $52(30.2)$ & $<0.0001$ \\
\hline $\mathrm{CHADS}_{2}$ score, median (IQR) & $3(2-4)$ & $3(2-4)$ & $2(2-4)$ & 0.0123 \\
\hline $\mathrm{CHA}_{2} \mathrm{DS}_{2}$-VASc score, median (IQR) & $4(3-5)$ & $5(3-6)$ & $4(3-5)$ & 0.0011 \\
\hline HAS-BLED score, median (IQR) & $3(2-3)$ & $3(2-3)$ & $4(3-4)$ & 0.6465 \\
\hline Preadmission mRS, median (IQR) & $0(0-2)$ & $0(0-2)$ & $0(0-2)$ & 0.4561 \\
\hline NIHSS score on admission, median (IQR) & $7(2-18)$ & $6(2-18)$ & $12(4-23)$ & $<0.0001$ \\
\hline \multicolumn{5}{|l|}{ Laboratory at admission } \\
\hline LDL, mg/dL, median (IQR) & $100(82-121)$ & $100(83-121)$ & $97(80-117)$ & 0.4083 \\
\hline Ccr, ml/min, median (IQR) & $53(37-71)$ & $52(36-68)$ & $58(43-83)$ & $<0.0001$ \\
\hline Blood glucose, mg/dL, median (IQR) & $126(108-154)$ & $126(108-152)$ & $131(109-159)$ & 0.1136 \\
\hline HbA1c, $(\%)$, median (IQR) & $6.0(5.6-6.4)$ & $6.0(5.7-6.4)$ & $5.9(5.5-6.3)$ & 0.0268 \\
\hline APTT, sec, median (IQR) & $32(28-37)$ & $32(28-37)$ & $33(29-38)$ & 0.0254 \\
\hline PT-INR, median (IQR) & $1.21(1.08-1.50)$ & $1.21(1.08-1.48)$ & $1.23(1.08-1.81)$ & 0.1242 \\
\hline High D-dimer*, n $(\%)$ & $439(50.6)$ & $384(55.0)$ & $55(32.4)$ & $<0.0001$ \\
\hline High BNP/NT-proBNP ${ }^{\dagger}, \mathrm{n}(\%)$ & $646(77.9)$ & $543(80.3)$ & $103(67.3)$ & 0.0005 \\
\hline Cerebral microbleeds, $\mathrm{n}(\%)$ & $221(31.3)$ & $149(26.0)$ & $72(54.1)$ & $<0.0001$ \\
\hline $\mathrm{mRS}$ at discharge, median (IQR) & $3(1-5)$ & $3(1-4)$ & $4(3-5)$ & $<0.0001$ \\
\hline Mortality during hospitalization, $\mathrm{n}(\%)$ & $57(6.4)$ & $36(5.0)$ & 21 (11.6) & 0.0012 \\
\hline
\end{tabular}

aPTT: activated partial thromboplastin time, BNP: brain natriuretic peptide, BP: blood pressure, Ccr: Creatinine clearance, ICH: Intracerebral hemorrhage, IQR: interquartile range, IS: Ischemic stroke, LDL: low-density lipoprotein cholesterol, mRS: modified Rankin Scale, NIHSS: National Institutes of Health stroke scale, NOAC: non-vitamin K antagonists, NT-proBNP: N-terminal B-type natriuretic peptide, PT-INR: prothrombin time-international normalized ratio, T-cho: total cholesterol, TG: triglycerides, TIA: Transient ischemic attack, VKA: vitamin K antagonist. High D-dimer* was defined as $1.0 \mu \mathrm{g} / \mathrm{mL}$ or more. High $\mathrm{BNP}$ or NT-proBNP ${ }^{\dagger}$ was defined as $>100$ or $>300 \mathrm{pg} / \mathrm{mL}$, respectively.

ICH, while high BNP/NT-proBNP (OR, 1.69; 95\% CI, 1.012.80; $\mathrm{p}=0.0486)$ and high $\mathrm{D}$-dimer levels (OR, 2.38; 95\% CI, 1.41-4.02; $\mathrm{p}=0.0010$ ) were independently associated with IS/TIA.

\section{Differences in clinical characteristics according to prior NOAC and VKA use}

Table 3 presents the clinical characteristics of patients in both groups according to prior NOAC and VKA use, while Fig. 2 illustrates the distribution of IS/TIA and ICH patients according to the clinical characteristics. Among prior NOAC users, ICH patients were younger than IS/TIA patients (Fig. 2A; $\mathrm{p}=0.0073$ ). Furthermore, a history of $\mathrm{ICH}$, poor pre-stroke BP control, CMBs, and high Ccr (Fig. 2E) were more common among ICH patients than among IS/TIA patients $(\mathrm{p}=0.0003, \mathrm{p}<0.0001, \mathrm{p}<0.0001$, and $\mathrm{p}=0.0200$, respectively). Although the $\mathrm{CHADS}_{2}, \mathrm{CHA}_{2} \mathrm{DS}_{2}$-VASc (Fig. 2B), and HAS-BLED scores (Fig. 2C) as well as APTT and PT-INR (Fig. 2D) did not significantly differ between the groups (all $\mathrm{p}>0.05$ ), the BNP/NT-proBNP and Ddimer levels were higher among IS/TIA patients than among ICH patients $(p=0.0042$ and $p=0.0008$, respectively). Stroke 
Table 2. Multivariable Logistic Regression Analysis for the Development of Intracerebral Hemorrhage.

\begin{tabular}{lccc}
\hline \multicolumn{1}{c}{ Variables } & OR & $95 \%$ CI & p value \\
\hline Age (per 10 years) & 0.80 & $0.57-1.13$ & 0.2052 \\
Female gender & 0.75 & $0.44-1.29$ & 0.3024 \\
NOAC plus APT & 1.28 & $0.79-2.07$ & 0.3249 \\
Previous ICH & 4.03 & $1.64-9.92$ & 0.0024 \\
Poor BP control prior to admission & 1.34 & $0.75-2.32$ & 0.2988 \\
Alcohol & 1.42 & $0.79-2.86$ & 0.2319 \\
Abnormal liver fnction & 1.74 & $0.51-5.97$ & 0.3773 \\
Ccr (per 10) & 1.04 & $0.93-1.18$ & 0.4820 \\
HbA1c (per 1) & 0.83 & $0.60-1.11$ & 0.2139 \\
APTT (per 1) & 1.02 & $1.00-1.04$ & 0.0317 \\
High D-dimer* & 0.42 & $0.25-0.71$ & 0.0010 \\
High BNP/NT-proBNP & 0.59 & $0.35-0.99$ & 0.0486 \\
Cerebral microbleeds & 3.64 & $2.25-5.87$ & $<0.0001$ \\
\hline
\end{tabular}

BNP: brain natriuretic peptide, BP: blood pressure, CI confidence interval, Ccr: Creatinine clearance, ICH: Intracerebral hemorrhage, IS: Ischemic stroke, NT-proBNP: N-terminal B-type natriuretic peptide, OR: odds ratio, PT-INR: prothrombin time-international normalized ratio. TIA: Transient ischemic attack. High D-dimer* was defined as $1.0 \mu \mathrm{g} / \mathrm{mL}$ or more. High BNP or NT-proBNP ${ }^{\dagger}$ was defined as $>100$ or $>300 \mathrm{pg} / \mathrm{mL}$, respectively.

severity, including the NIHSS score on admission $(\mathrm{p}<$ $0.0001)$, the mRS score on discharge $(\mathrm{p}<0.0001)$, and mortality $(\mathrm{p}=0.0043)$ during hospitalization were higher in the ICH group than in the IS/TIA group.

Among prior VKA users, IS/TIA patients were older than ICH patients (Fig. 2A; $\mathrm{p}=0.0005$ ). Furthermore, a history of IS/TIA and high D-dimer levels $(\mathrm{p}=0.0020$ and $\mathrm{p}<0.0001$, respectively) as well as high $\mathrm{CHADS}_{2}$ and $\mathrm{CHA}_{2} \mathrm{DS}_{2}-\mathrm{VASc}$ scores (Fig. 2B) ( $p=0.0001$ and $p<0.0001$, respectively) were significantly more common among IS/TIA patients than among ICH patients. However, the incidence of previous ICH and alcohol use were higher among ICH patients than among IS/TIA patients $(\mathrm{p}<0.0001$ and $\mathrm{p}=0.0125$, respectively). The HAS-BLED score did not differ markedly between the groups (Fig. 2C; p=0.1763). The Ccr, APTT, and PT-INR were significantly higher among ICH patients than among IS/TIA patients $(\mathrm{p}=0.0023, \mathrm{p}<0.0001$, and $\mathrm{p}<0.0001$, respectively). A PT-INR of $<1.6$ was more common among IS/TIA patients, whereas a PT-INR of 2.0-2.5 was more common among ICH patients (Fig. 2D). There were no significant differences in the proportion of the presence of CMBs (Fig. 2E) between the IS/TIA and ICH groups ( $\mathrm{p}=$ 0.2397 and $\mathrm{p}=0.2212$, respectively). The level of stroke severity, including the discharge mRS score $(\mathrm{p}=0.0333)$, and the mortality rate during hospitalization $(p=0.0477)$ but not the NIHSS score on admission $(\mathrm{p}=0.3268)$ were higher in the ICH group than in the IS/TIA group.

\section{Factors associated with ICH or ISITIA according to prior NOAC and VKA use}

Table 4 presents the findings of the multivariable logistic regression analysis of predictors of $\mathrm{ICH}$ according to prior $\mathrm{OAC}$ use. Among prior NOAC users, the occurrence of
CMBs (OR, 4.77; 95\% CI, 2.69-8.47; p<0.0001) was independently associated with ICH. Furthermore, high BNP/NTproBNP levels (OR, 1.89; 95\% CI, 1.03-3.45; p=0.0390) were independently associated with IS/TIA. Among prior VKA users, a history of ICH (OR, 13.59; 95\% CI, 1.33139.17; $\mathrm{p}=0.0279)$ and a high PT-INR (OR, 1.17; 95\% CI, $1.10-1.26 ; \mathrm{p}<0.0001)$ were independently associated with ICH, while a history of IS/TIA (OR, 3.37; 95\% CI, 1.348.49; $\mathrm{p}=0.0101)$ and high D-dimer levels (OR, 2.47; 95\% CI, 1.05-5.82; $\mathrm{p}=0.0377$ ) were independently associated with IS/TIA.

\section{Discussion}

This study yielded several major findings. First, during the study period, NOACs were prescribed to more than $60 \%$ of patients with both IS/TIA and ICH during OAC therapy. Second, although the prevalence of cardiovascular risk factors was similar between IS/TIA and ICH, the presence of CMBs was independently associated with $\mathrm{ICH}$, and high BNP/NT-proBNP levels were independently associated with IS/TIA among NOAC users. Third, among VKA users, a history of ICH and the PT-INR were independently associated with ICH, while a history of IS/TIA and high D-dimer levels were independently associated with IS/TIA.

In the present study, the presence of CMBs was independently associated with ICH among prior NOAC users. A previous meta-analysis of 15 prospective studies, including patients with IS or TIA, suggested a higher risk of future ICH than IS in patients with CMBs (28). Recently, an observational cohort study in Europe, similar to our study, found that patients with NOAC-related ICH are more likely to have more CMBs than patients with NOAC-related IS; however, the sample of that study was relatively small $(n=116)$. A retrospective cohort study suggested that NOACs might trigger ICH only in patients at particularly high risk of $\mathrm{ICH}$, such as those with $\mathrm{CMBs}$ and high small-vessel disease scores $(18,29)$. However, in our study, we did not systematically collect data on the location of the CMBs. Further studies are therefore needed to ascertain how best to manage patients with CMBs who require OACs and to determine the characteristics of patients in whom OACs should be prescribed or avoided.

We found that high D-dimer levels among patients receiving VKAs and high BNP/NT-proBNP levels among patients receiving NOACs were independently associated with IS/ TIA. Previous reports suggest that elevated D-dimer levels are common in patients with $\mathrm{AF}$ and are an additional risk factor for stroke. D-dimer levels are suppressed by anticoagulant therapy, but even in patients receiving oral anticoagulation, D-dimer levels can independently predict stroke or systemic embolism, cardiovascular mortality, and bleeding (30-32). These present and previous findings suggest that D-dimer levels may also be a clinically useful risk marker of IS in AF during OAC therapy. A pooled data meta-analysis demonstrated increased BNP/NT-proBNP lev- 
Table 3. Comparison of Clinical Background Characteristics according to Prior Direct Oral Anticoagulant or Warfarin Use.

\begin{tabular}{|c|c|c|c|c|c|c|}
\hline \multirow[t]{2}{*}{ Variable } & \multicolumn{3}{|c|}{ NOAC } & \multicolumn{3}{|c|}{ VKA } \\
\hline & IS/TIA n=462 & $\mathrm{ICH} n=132$ & $\mathrm{p}$ value & IS/TIA n=253 & $\mathrm{ICH} n=49$ & $\mathrm{p}$ value \\
\hline Age, years, median (IQR) & $79(73-85)$ & $77(70-82)$ & 0.0073 & $81(76-85)$ & $75(70-83)$ & 0.0005 \\
\hline Female gender, n $(\%)$ & $177(38.3)$ & $44(33.3)$ & 0.2967 & $110(43.4)$ & $14(28.6)$ & 0.0522 \\
\hline \multicolumn{7}{|l|}{ Risk factors, n (\%) } \\
\hline Previous IS/TIA & $202(43.7)$ & $58(43.9)$ & 0.9647 & $117(46.3)$ & $11(22.5)$ & 0.0020 \\
\hline Previous ICH & $12(2.6)$ & $13(9.9)$ & 0.0003 & $2(0.8)$ & $6(12.2)$ & $<0.0001$ \\
\hline Hypertension & 368 (79.7) & $114(86.4)$ & 0.0822 & $201(79.5)$ & $38(77.6)$ & 0.7650 \\
\hline Diabetes mellitus & $134(29.0)$ & $30(22.7)$ & 0.1548 & $67(26.5)$ & $12(24.5)$ & 0.7715 \\
\hline Congestive heart failure & $116(25.1)$ & $33(25.0)$ & 0.9798 & $86(34.0)$ & $13(26.5)$ & 0.3085 \\
\hline Dyslipidemia & $164(35.5)$ & $49(37.1)$ & 0.7316 & $96(37.9)$ & $14(28.6)$ & 0.2120 \\
\hline Smoking & $118(25.5)$ & $30(22.7)$ & 0.5098 & $50(19.8)$ & $11(22.5)$ & 0.6682 \\
\hline Alcohol & $89(19.3)$ & $32(24.2)$ & 0.2104 & $32(12.7)$ & $13(26.5)$ & 0.0125 \\
\hline History of vascular disease, $\mathrm{n}(\%)$ & $62(13.4)$ & $16(12.1)$ & 0.6968 & $41(16.2)$ & $10(20.4)$ & 0.4723 \\
\hline Abnormal renal function, n (\%) & $4(0.9)$ & $1(0.8)$ & 0.9045 & $14(5.5)$ & $5(10.2)$ & 0.2178 \\
\hline Abnormal liver function, $\mathrm{n}(\%)$ & $11(2.4)$ & $7(5.3)$ & 0.0841 & $5(2.0)$ & $2(4.1)$ & 0.3700 \\
\hline Poor BP control prior to admission, $\mathrm{n}(\%)$ & $78(17.1)$ & $42(33.1)$ & $<0.0001$ & $41(16.6)$ & $10(22.2)$ & 0.3609 \\
\hline $\mathrm{CHADS}_{2}$ score, median (IQR) & $3(2-4)$ & $3(2-4)$ & 0.6307 & $3(2-4)$ & $2(1-3)$ & 0.0001 \\
\hline $\mathrm{CHA}_{2} \mathrm{DS}_{2}$-VASc score, median (IQR) & $4(3-6)$ & $4(3-5)$ & 0.2423 & $5(4-6)$ & $3(3-5)$ & $<0.0001$ \\
\hline HAS-BLED score, median (IQR) & $3(2-3)$ & $3(2-3)$ & 0.0532 & $3(2-4)$ & $3(3-5)$ & 0.1763 \\
\hline Concomitant use of antiplatelet therapy, $\mathrm{n}(\%)$ & $60(13.0)$ & $24(18.2)$ & 0.1309 & $59(23.3)$ & $10(20.4)$ & 0.6568 \\
\hline Preadmission mRS, median (IQR) & $0(0-2)$ & $0(0-2)$ & 0.6827 & $1(0-3)$ & $0(0-2)$ & 0.0877 \\
\hline NIHSS score on admission, median (IQR) & $5(2-16)$ & $11(4-23)$ & $<0.0001$ & $8(3-21)$ & $13(4-22)$ & 0.3268 \\
\hline \multicolumn{7}{|l|}{ Biochemistry sign at admission } \\
\hline LDL, mg/dL, median (IQR) & $100(84-122)$ & $97(79-116)$ & 0.2358 & $100(80-118)$ & $97(82-120)$ & 0.7492 \\
\hline Ccr, ml/min, median (IQR) & $55(39-72)$ & $57(43-84)$ & 0.0200 & $44(32-63)$ & $59(37-77)$ & 0.0023 \\
\hline Blood glucose, mg/dL, median (IQR) & $128(109-155)$ & $133(114-157)$ & 0.2471 & $123(105-149)$ & $127(103-166)$ & 0.4550 \\
\hline HbA1c, $(\%)$, median (IQR) & $6.0(5.7-6.4)$ & $5.9(5.5-6.3)$ & 0.0286 & $6.0(5.6-6.4)$ & $5.9(5.5-6.2)$ & 0.4004 \\
\hline APTT, sec, median (IQR) & $32(28-36)$ & $32(28-36)$ & 0.6415 & $32(28-37)$ & $38(34-45)$ & $<0.0001$ \\
\hline PT-INR, median (IQR) & $1.14(1.04-1.30)$ & $1.14(1.05-1.29)$ & 0.8983 & $1.43(1.23-1.83)$ & $2.23(1.84-2.71)$ & $<0.0001$ \\
\hline High D-dimer*, n (\%) & $227(50.4)$ & $41(33.3)$ & 0.0008 & $157(63.3)$ & $14(29.8)$ & $<0.0001$ \\
\hline High BNP/NT-proBNP ${ }^{\dagger}, \mathrm{n}(\%)$ & $337(77.5)$ & $72(64.3)$ & 0.0042 & $206(85.5)$ & $31(75.6)$ & 0.1107 \\
\hline Cerebral microbleeds, n (\%) & $95(25.1)$ & $58(60.4)$ & $<0.0001$ & $54(27.8)$ & $14(37.8)$ & 0.2212 \\
\hline $\mathrm{mRS}$ at discharge, median (IQR) & $3(1-4)$ & $4(3-5)$ & $<0.0001$ & $4(1-5)$ & $4(3-5)$ & 0.0333 \\
\hline Mortality during hospitalization, $\mathrm{n}(\%)$ & $17(3.7)$ & $13(9.9)$ & 0.0043 & $19(7.5)$ & $8(16.3)$ & 0.0477 \\
\hline
\end{tabular}

aPTT: activated partial thromboplastin time, BNP: brain natriuretic peptide, BP: blood pressure, Ccr: Creatinine clearance, ICH: Intracerebral hemorrhage, IQR: interquartile range, IS: Ischemic stroke, LDL: low-density lipoprotein cholesterol, mRS: modified Rankin Scale, NIHSS: National Institutes of Health stroke scale, NOAC: non-vitamin K antagonist, NT-proBNP: N-terminal B-type natriuretic peptide, PT-INR: prothrombin time-international normalized ratio, T-cho: total cholesterol, TG: triglycerides, TIA: Transient ischemic attack, VKA: vitamin K antagonist. High D-dimer* was defined as $1.0 \mu \mathrm{g} / \mathrm{mL}$ or more. High BNP or NT-proBNP ${ }^{\dagger}$ was defined as $>100$ or $>300 \mathrm{pg} / \mathrm{mL}$, respectively.

els in patients with cardioembolic stroke (33). A previous prospective study also showed that the plasma BNP level was significantly higher in the acute phase of stroke than in the subacute phase, suggesting that heart failure may be associated with the onset of IS in patients with AF (34). Although the levels of D-dimer and BNP/NT-pro BNP on admission may be influenced by stroke itself and other concomitant confounders, the present study suggests that combined strategies for managing modifiable factors, such as coagulation and heart failure, may be effective for preventing stroke in patients receiving OACs.

Among patients receiving VKAs, the PT-INR was associated with ICH. A previous study identified Asian ethnicity as a risk factor for VKA-associated $\mathrm{ICH}$, which may be partly attributed to genetic differences affecting VKA me- tabolism or the treatment response (35-37). Unlike in western countries, the Japanese domestic guidelines recommend a PT-INR value of 1.6-2.6 for patients with non-valvular AF $(38,39)$. Unlike NOAC users, there was no significant difference in the presence of CMBs between IS/TIA and ICH patients among VKA users. A systematic review and meta-analysis suggested that $\mathrm{CMBs}$ are associated with an increased risk of future $\mathrm{ICH}$, particularly in patients receiving VKAs (40). This may be because physicians tend to choose NOACs over VKAs for patients with a high ICH risk, such as those with CMBs. Another possible reason is that VKAs may be used while keeping PT-INR low in patients with $\mathrm{CMB}$ and a history of $\mathrm{ICH}$, which may increase the IS and diminish the impact of the presence of CMBs on VKA-related ICH. Furthermore, there may be differences in 

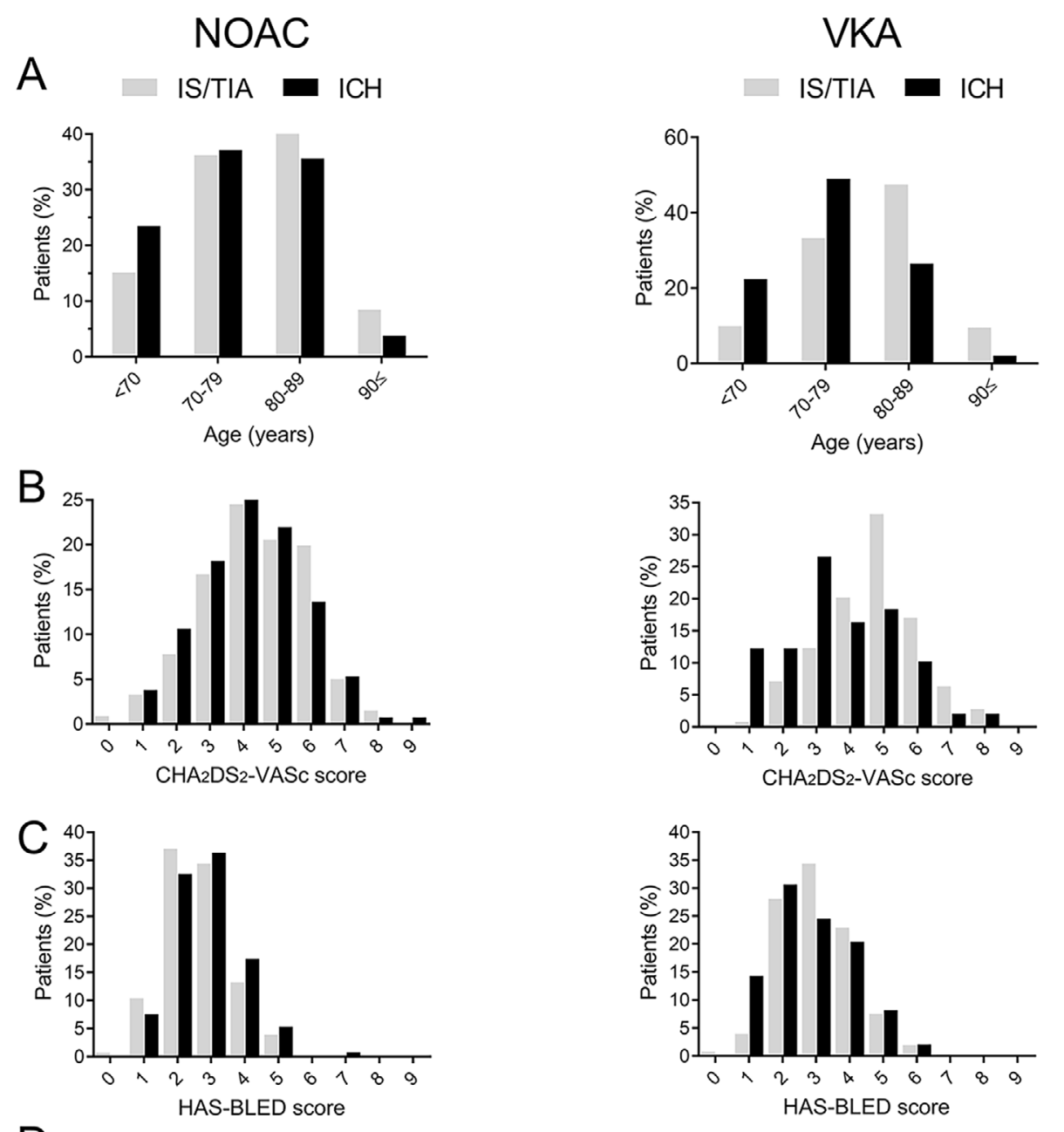

D
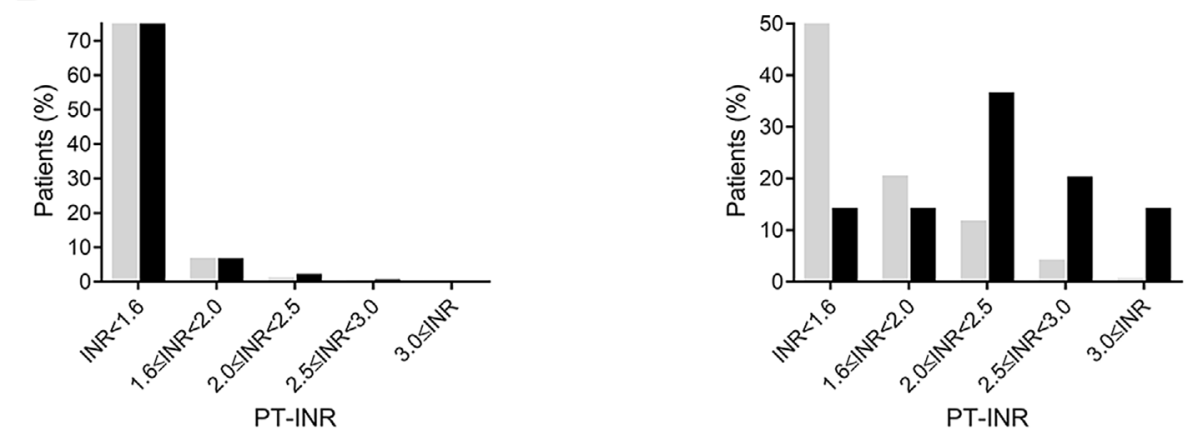

$E$
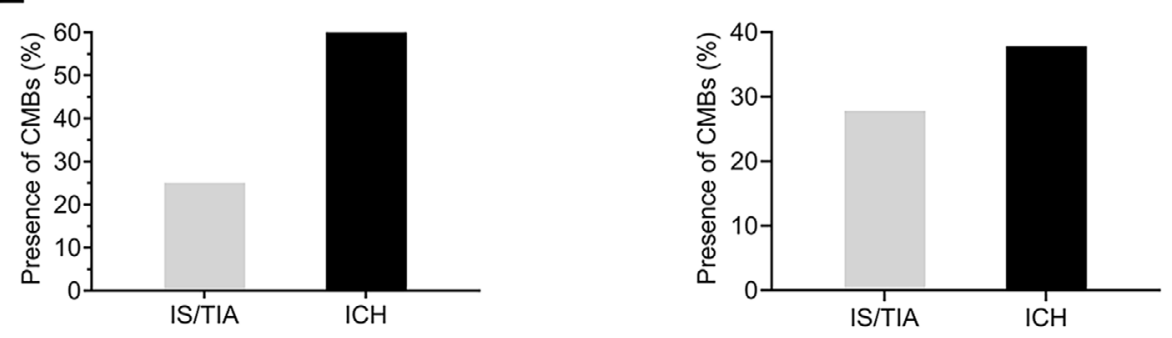

Figure 2. Distribution of patients according to clinical characteristics. Patients were stratified based on (A) age, (B) CHA CS $_{2}$-VASc scores, (C) HAS-BLED scores, (D) PT-INR, and (E) CMBs according to the prior use of non-vitamin $\mathrm{K}$ oral antagonists (NOACs) and vitamin $\mathrm{K}$ antagonists (VKAs). CMBs: cerebral microbleeds, ICH: intracerebral hemorrhage, IS: ischemic stroke, PT-INR: prothrombin time-international normalized ratio, TIA: transient ischemic attack 
Table 4. Multivariable Logistic Regression Analysis for the Development of Intracerebral Hemorrhage according to Prior NOAC or VKA Use.

\begin{tabular}{|c|c|c|c|c|c|c|}
\hline \multirow[b]{2}{*}{ Variables } & \multicolumn{3}{|c|}{ Prior NOAC prescription cohort } & \multicolumn{3}{|c|}{ Prior VKA prescription cohort } \\
\hline & OR & $95 \% \mathrm{CI}$ & $\mathrm{p}$ value & OR & $95 \% \mathrm{CI}$ & $\mathrm{p}$ value \\
\hline Age (per 10 years) & 0.78 & $0.52-1.17$ & 0.2270 & 0.65 & $0.39-1.08$ & 0.0986 \\
\hline Female gender & 0.81 & $0.44-1.48$ & 0.4968 & 0.56 & $0.21-1.49$ & 0.2463 \\
\hline Previous IS/TIA & - & - & - & 0.30 & $0.12-0.75$ & 0.0101 \\
\hline Previous ICH & 2.11 & $0.76-5.90$ & 0.1521 & 13.59 & $1.33-139.17$ & 0.0279 \\
\hline Alcohol & - & - & - & 1.30 & $0.49-3.46$ & 0.5957 \\
\hline Poor BP control prior to admission & 1.36 & $0.72-2.58$ & 0.3456 & 1.58 & $0.61-4.11$ & 0.3497 \\
\hline Ccr $($ per 10$)$ & 1.00 & $0.87-1.16$ & 0.9672 & - & - & - \\
\hline HbA1c (per 1) & 0.31 & $0.57-1.14$ & 0.2552 & - & - & - \\
\hline PT-INR (per 0.1) & - & - & - & 1.17 & $1.10-1.26$ & $<0.0001$ \\
\hline High D-dimer* & 0.56 & $0.31-1.01$ & 0.0540 & 0.40 & $0.17-0.95$ & 0.0377 \\
\hline High BNP/NT-proBNP ${ }^{\dagger}$ & 0.53 & $0.29-0.97$ & 0.0390 & - & - & - \\
\hline Cerebral microbleeds & 4.77 & $2.69-8.47$ & $<0.0001$ & - & - & - \\
\hline
\end{tabular}

BNP: brain natriuretic peptide, BP: blood pressure, CI confidence interval, Ccr: Creatinine clearance, ICH: Intracerebral hemorrhage, IS: Ischemic stroke, NOAC: non-vitamin K antagonists, NT-proBNP: N-terminal B-type natriuretic peptide, OR: odds ratio, PT-INR: prothrombin time-international normalized ratio. TIA: Transient ischemic attack, VKA: vitamin

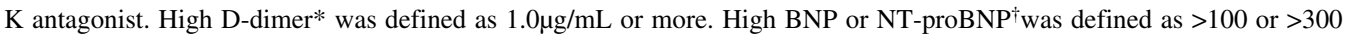
$\mathrm{pg} / \mathrm{mL}$, respectively.

the strictness of blood pressure control prior to stroke between patients on NOACs and VKAs. As we have no data on the detailed location and number of CMBs and duration of OAC medication, longitudinal prospective studies are needed to confirm the relationship between development of new CMB/ICH and OAC treatment.

Several limitations associated with the present study warrant mention. Due to the cross-sectional design and the fact that nearly half of the patients in this cohort had a history of stroke, there was potential selection bias, and we merely compared ICH and IS/TIA rather than demonstrating a causative relationship. Thus, firm conclusions regarding the absolute risk factors cannot be drawn. A recent analysis of the National Health Insurance Database from the Tsugaru region of Aomori Prefecture in Japan showed that among $\mathrm{AF}$ patients on OAC, 32\% were on warfarin in 2016 and $27 \%$ in 2017 (41). In the present study, the proportion of AF patients prescribed warfarin was $34 \%$, which is relatively high. This may be explained by two possible reasons: (1) patients that had been diagnosed with AF before 2011 were started on VKA and did not switch to NOACs, and (2) patients taking warfarin were more likely to develop stroke than those taking NOACs. Unfortunately, we were unable to gather data regarding the pre-stroke duration of $\mathrm{AF}$ burden, OAC therapy, or time since the last stroke. Finally, almost all enrolled patients were Japanese; thus, the results of this study may not be generalizable to all ethnicities.

Nevertheless, our study has certain strengths, including a multicenter setting, a relatively large sample size, and the analysis of combined ischemic and hemorrhagic stroke data.

\section{Conclusion}

Our findings suggest that the presence of CMBs and na- triuretic peptides may be useful for risk stratification of either IS or ICH development in patients receiving NOACs. In contrast, a history of stroke, the PT-INR, and D-dimer levels may be useful for risk stratification of either IS or ICH development in patients receiving VKAs. Further longitudinal studies and validation of these findings in other cohorts are required to investigate the role of a stroke history, neuroimaging, and cardiac and coagulation laboratory markers in the selection or management of patients regarding OAC therapy.

\section{Author's disclosure of potential Conflicts of Interest (COI).}

Satoshi Suda: Honoraria, Eisai Co., Ltd.; Research funding, the All Japan Coffee Association. Yasuyuki Iguchi: Honoraria, Sanofi Co., Ltd., Daiichi-Sankyo Co., Ltd, Nippon Boehringer Ingelheim Co. Ltd, Bayer Healthcare Co. Ltd, Pfizer Inc Co. Ltd. and Bristol-Myers Squibb Co. Ltd. Yoshiki Yagita: Honoraria, Daiichi-Sankyo Co., Ltd. Takao Kanzawa: Honoraria, DaiichiSankyo Co., Ltd. Shigeru Fujimoto: Honoraria, Takeda Pharmaceutical Co., Ltd., Bayer Yakuhin, Co., Ltd. and Daiichi-Sankyo Co., Ltd. Makoto Nakajima: Honoraria, Daiichi-Sankyo Co. Ltd. Takehiko Nagao: Honoraria, Bayer Yakuhin, Co., Ltd. Kazumi Kimura: Honoraria, Bristol-Myers Squibb Co. Ltd., Nippon Boehringer Ingelheim Co. Ltd., Bayer Healthcare Co. Ltd. and Daiichi Sankyo Co. Ltd.; Research funding, Nippon Boehringer Ingelheim Co. Ltd. and Daiichi Sankyo Co. Ltd. Yu Kono: Research funding, Sanofi Co., Ltd.

\section{Acknowledgement}

We thank the following PASTA investigators and their hospitals for participating in this study: Rinko Kokubo, Tomoyuki Kono, Takafumi Mashiko, Hiroshi Okada, Naoki Oyama, Kenichiro Sakai, Tomonari Saito, Masayuki Suzuki, Kenichi Todo, and Masayuki Ueda. 


\section{Funding Sources}

This research was supported by Nippon Boehringer Ingelheim Co., Ltd.

\section{References}

1. Akao M, Chun YH, Wada H, et al. Current status of clinical background of patients with atrial fibrillation in a community-based survey: the Fushimi AF Registry. J Cardiol 61: 260-266, 2013.

2. Iguchi Y, Kimura K, Aoki J, et al. Prevalence of atrial fibrillation in community-dwelling Japanese aged 40 years or older in Japan: analysis of 41,436 non-employee residents in Kurashiki-city. Circ J 72: 909-913, 2008.

3. Kimura K, Minematsu K, Yamaguchi T; Japan Multicenter Stroke Investigators C. Atrial fibrillation as a predictive factor for severe stroke and early death in 15,831 patients with acute ischaemic stroke. J Neurol Neurosurg Psychiatry 76: 679-683, 2005.

4. Hylek EM, Evans-Molina C, Shea C, Henault LE, Regan S. Major Hemorrhage and Tolerability of Warfarin in the First Year of Therapy Among Elderly Patients With Atrial Fibrillation. Circulation 115: 2689-2696, 2007.

5. Ruff CT, Giugliano RP, Braunwald E, et al. Comparison of the efficacy and safety of new oral anticoagulants with warfarin in patients with atrial fibrillation: a meta-analysis of randomised trials. Lancet 383: 955-962, 2014.

6. Caldeira D, Rodrigues FB, Barra M, et al. Non-vitamin K antagonist oral anticoagulants and major bleeding-related fatality in patients with atrial fibrillation and venous thromboembolism: a systematic review and meta-analysis. Heart 101: 1204-1211, 2015.

7. Senoo K, Lau YC, Dzeshka M, Lane D, Okumura K, Lip GY. Efficacy and safety of non-vitamin $\mathrm{K}$ antagonist oral anticoagulants vs. warfarin in Japanese patients with atrial fibrillation - metaanalysis. Circ J 79: 339-345, 2015.

8. Hindricks G, Potpara T, Dagres N, et al. 2020 ESC Guidelines for the diagnosis and management of atrial fibrillation developed in collaboration with the European Association of Cardio-Thoracic Surgery (EACTS). Eur Heart J 42: 373-498, 2021.

9. January CT, Wann LS, Calkins H, et al. 2019 AHA/ACC/HRS Focused Update of the 2014 AHA/ACC/HRS Guideline for the Management of Patients With Atrial Fibrillation: A Report of the American College of Cardiology/American Heart Association Task Force on Clinical Practice Guidelines and the Heart Rhythm Society in Collaboration With the Society of Thoracic Surgeons. Circulation 140: e125-e151, 2019.

10. Toyoda K, Arihiro S, Todo K, et al. Trends in oral anticoagulant choice for acute stroke patients with nonvalvular atrial fibrillation in Japan: the SAMURAI-NVAF study. Int J Stroke 10: 836-842, 2015.

11. Lip GYH, Lane DA, Buller H, Apostolakis S. Development of a novel composite stroke and bleeding risk score in patients with atrial fibrillation: the AMADEUS Study. Chest 144: 1839-1847, 2013.

12. Okumura Y. What Scoring System Should We Use to Assess Bleeding Risk in Atrial Fibrillation? Circ J 80: 2089-2091, 2016.

13. Maruhashi T, Higashi Y. Antithrombotic therapy for stroke prevention in patients with atrial fibrillation in Japan. Expert Opin Pharmacother 21: 2115-2124, 2020.

14. Takahashi H, Jimbo Y, Takano H, et al. Intracerebral Hematoma Occurring During Warfarin Versus Non-Vitamin K Antagonist Oral Anticoagulant Therapy. Am J Cardiol 118: 222-225, 2016.

15. Auer E, Frey S, Kaesmacher J, et al. Stroke severity in patients with preceding direct oral anticoagulant therapy as compared to vitamin K antagonists. J Neurol 266: 2263-2272, 2019.

16. Sakamoto Y, Okubo S, Nito C, et al. The relationship between stroke severity and prior direct oral anticoagulant therapy in pa- tients with acute ischaemic stroke and non-valvular atrial fibrillation. Eur J Neurol 24: 1399-1406, 2017.

17. Kawabori M, Niiya Y, Iwasaki M, et al. Characteristics of Symptomatic Intracerebral Hemorrhage in Patient Receiving Direct Oral Anticoagulants: Comparison with Warfarin. J Stroke Cerebrovasc Dis 27: 1338-1342, 2018.

18. Suda S, Aoki J, Shimoyama T, et al. Characteristics of Acute Spontaneous Intracerebral Hemorrhage in Patients Receiving Oral Anticoagulants. J Stroke Cerebrovasc Dis 28: 1007-1014, 2019.

19. Suda S, Iguchi Y, Fujimoto S, et al. Multicenter Prospective Analysis of Stroke Patients Taking Oral Anticoagulants: The PASTA Registry - Study Design and Characteristics. J Stroke Cerebrovasc Dis 28: 104456, 2019.

20. European Heart Rhythm A, Camm AJ, et al.; European Association for Cardio-Thoracic S. Guidelines for the management of atrial fibrillation: the Task Force for the Management of Atrial Fibrillation of the European Society of Cardiology (ESC). Eur Heart J 31: 2369-2429, 2010.

21. Pisters R, Lane DA, Nieuwlaat R, de Vos CB, Crijns HJ, Lip GY. A novel user-friendly score (HAS-BLED) to assess 1-year risk of major bleeding in patients with atrial fibrillation: the Euro Heart Survey. Chest 138: 1093-1100, 2010.

22. Hamatani Y, Nagai T, Nakai M, et al. Elevated Plasma D-Dimer Level Is Associated With Short-Term Risk of Ischemic Stroke in Patients With Acute Heart Failure. Stroke 49: 1737-1740, 2018.

23. Yoshimuta $T$, Yokoyama $H$, Okajima $T$, et al. Impact of Elevated D-Dimer on Diagnosis of Acute Aortic Dissection With Isolated Neurological Symptoms in Ischemic Stroke. Circ J 79: 1841-1845, 2015.

24. Ohara T, Koga M, Tokuda N, et al. Rapid Identification of Type A Aortic Dissection as a Cause of Acute Ischemic Stroke. J Stroke Cerebrovasc Dis 25: 1901-1906, 2016.

25. Ponikowski P, Voors AA, Anker SD, et al. 2016 ESC Guidelines for the diagnosis and treatment of acute and chronic heart failure: The Task Force for the diagnosis and treatment of acute and chronic heart failure of the European Society of Cardiology (ESC). Developed with the special contribution of the Heart Failure Association (HFA) of the ESC. Eur J Heart Fail 18: 891-975, 2016.

26. Todo K, Iwata T, Doijiri R, et al. Frequent Premature Atrial Contractions in Cryptogenic Stroke Predict Atrial Fibrillation Detection with Insertable Cardiac Monitoring. Cerebrovasc Dis 49: 144$150,2020$.

27. Suda S, Shimoyama T, Suzuki S, et al. Prevalence and clinical characteristics of cortical superficial siderosis in patients with acute stroke. J Neurol 264: 2413-2419, 2017.

28. Wilson D, Charidimou A, Ambler G, et al. Recurrent stroke risk and cerebral microbleed burden in ischemic stroke and TIA: A meta-analysis. Neurology 87: 1501-1510, 2016.

29. Hagii J, Tomita H, Metoki N, et al. Characteristics of intracerebral hemorrhage during rivaroxaban treatment: comparison with those during warfarin. Stroke 45: 2805-2807, 2014.

30. Choi KH, Seo WK, Park MS, et al. Baseline D-Dimer Levels as a Risk Assessment Biomarker for Recurrent Stroke in Patients with Combined Atrial Fibrillation and Atherosclerosis. J Clin Med 8: 2019.

31. Siegbahn A, Oldgren J, Andersson U, et al. D-dimer and factor VIIa in atrial fibrillation - prognostic values for cardiovascular events and effects of anticoagulation therapy. A RE-LY substudy. Thromb Haemost 115: 921-930, 2016.

32. Christersson C, Wallentin L, Andersson U, et al. D-dimer and risk of thromboembolic and bleeding events in patients with atrial fibrillation--observations from the ARISTOTLE trial. J Thromb Haemost 12: 1401-1412, 2014.

33. Llombart V, Antolin-Fontes A, Bustamante A, et al. B-type natriuretic peptides help in cardioembolic stroke diagnosis: pooled data 
meta-analysis. Stroke 46: 1187-1195, 2015.

34. Shibazaki K, Kimura K, Okada Y, Iguchi Y, Terasawa Y, Aoki J. Heart failure may be associated with the onset of ischemic stroke with atrial fibrillation: a brain natriuretic peptide study. J Neurol Sci 281: 55-57, 2009.

35. Rieder MJ, Reiner AP, Gage BF, et al. Effect of VKORC1 haplotypes on transcriptional regulation and warfarin dose. $\mathrm{N}$ Engl $\mathrm{J}$ Med 352: 2285-2293, 2005.

36. Takahashi H, Wilkinson GR, Caraco Y, et al. Population differences in S-warfarin metabolism between CYP2C9 genotypematched Caucasian and Japanese patients. Clin Pharmacol Ther 73: 253-263, 2003.

37. Shen AY, Yao JF, Brar SS, Jorgensen MB, Chen W. Racial/ethnic differences in the risk of intracranial hemorrhage among patients with atrial fibrillation. J Am Coll Cardiol 50: 309-315, 2007.

38. Shinohara Y. For readers (stroke specialists and general practitioners) of the Japanese guidelines for the management of stroke. Preface. J Stroke Cerebrovasc Dis 20: S1-S6, 2011.
39. Yasaka M, Minematsu K, Yamaguchi T. Optimal intensity of international normalized ratio in warfarin therapy for secondary prevention of stroke in patients with non-valvular atrial fibrillation. Intern Med 40: 1183-1188, 2001.

40. Charidimou A, Kakar P, Fox Z, Werring DJ. Cerebral microbleeds and recurrent stroke risk: systematic review and meta-analysis of prospective ischemic stroke and transient ischemic attack cohorts. Stroke 44: 995-1001, 2013.

41. Narita N, Okumura K, Kinjo $T$, et al. Trends in Prevalence of Non-Valvular Atrial Fibrillation and Anticoagulation Therapy in a Japanese Region - Analysis Using the National Health Insurance Database. Circ J 84: 706-713, 2020.

The Internal Medicine is an Open Access journal distributed under the Creative Commons Attribution-NonCommercial-NoDerivatives 4.0 International License. To view the details of this license, please visit (https://creativecommons.org/licenses/ by-nc-nd/4.0/).

(C) The Japanese Society of Internal Medicine Intern Med Advance Publication 